\title{
THE LEECH EXOPLANET IMAGING SURVEY: CHARACTERIZATION OF THE COLDEST DIRECTLY IMAGED EXOPLANET, GJ 504 b, AND EVIDENCE FOR SUPERSTELLAR METALLICITY*
}

\author{
Andrew J. Skemer ${ }^{1,2}$, Caroline V. Morley ${ }^{2}$, Neil T. Zimmerman ${ }^{3,4,5}$, Michael F. Skrutskie ${ }^{6}$ Jarron Leisenring $^{1}$, \\ Esther Buenzli ${ }^{3}$, Mickael Bonnefoy ${ }^{3,7}$, Vanessa Bailey ${ }^{1}$, Philip Hinz ${ }^{1}$, Denis Defrére ${ }^{1}$, Simone Esposito ${ }^{8}$, \\ Dániel Apai ${ }^{1,9}$, Beth Biller ${ }^{3,10}$, Wolfgang Brandner ${ }^{3}$, Laird Close ${ }^{1}$, Justin R. Crepp ${ }^{11}$, Robert J. De Rosa ${ }^{12,13}$, \\ Silvano Desidera ${ }^{14}$, Josh Eisner ${ }^{1}$, Jonathan Fortney ${ }^{2}$, Richard Freedman ${ }^{15,16}$, Thomas Henning ${ }^{3}$, \\ Karl-Heinz Hofmann $^{17}$, Taisiya Kopytova ${ }^{3}$, Roxana Lupu ${ }^{16}$, Anne-Lise Maire ${ }^{14}$, Jared R. Males ${ }^{1}$, Mark Marley ${ }^{16}$, \\ Katie Morzinski ${ }^{1}$, Apurva Oza ${ }^{6,18}$, Jenny Patience ${ }^{12}$, Abhijith Rajan ${ }^{12}$, George Rieke ${ }^{1}$, Dieter Schertl ${ }^{17}$, \\ Joshua Schlieder ${ }^{3,16}$, Jordan Stone ${ }^{1}$, Kate Su ${ }^{1}$, Amali Vaz ${ }^{1}$, Channon Visscher ${ }^{19}$, Kimberly Ward-DuOng ${ }^{12}$, \\ Gerd WeIGElT ${ }^{17}$, AND Charles E. WOODWARD ${ }^{20}$ \\ ${ }^{1}$ Steward Observatory, University of Arizona, 933 North Cherry Ave. Tucson, AZ 85721, USA \\ ${ }^{2}$ University of California, Santa Cruz, 1156 High St. Santa Cruz, CA 95064, USA \\ ${ }^{3}$ Max Planck Institute for Astronomy, Königstuhl 17, 69117 Heidelberg, Germany \\ ${ }^{4}$ Princeton University, 4 Ivy Ln, Princeton, NJ 08544, USA \\ ${ }^{5}$ Space Telescope Science Institute, 3700 San Martin Drive, Baltimore MD 21218, USA \\ ${ }^{6}$ University of Virginia, 530 McCormick Rd., Charlottesville, VA 22904, USA \\ ${ }_{8}^{7}$ Institut de Planetologie et d'Astrophysique de Grenoble, 414 Rue de la Piscine, 38400 St-Martin d'Heres, France \\ ${ }^{8}$ Istituto Nazionale di Astrofisica-Arcetri Astrophysical Observatory, Largo Enrico Fermi 5, 50125, Florence, Italy \\ ${ }^{9}$ Lunar and Planetary Laboratory, University of Arizona, 1629 East University Blvd, Tucson, AZ 85721, USA \\ ${ }^{10}$ University of Edinburgh, Old College, South Bridge, Edinburgh Eh8 9YL, UK \\ ${ }^{11}$ Notre Dame University, 225 Nieuwland Science Hall, Notre Dame, IN 46556, USA \\ ${ }_{12}$ Arizona State University, 781 South Terrace Rd, Tempe, AZ 85281, USA \\ ${ }^{13}$ University of California, Berkeley, 501 Campbell Hall, Berkeley, CA 94720, USA \\ ${ }^{14}$ Istituto Nazionale di Astrofisica-Padova Astronomical Observatory, Vicolo dell'Osservatorio 5, 35122 Padova, Italy \\ ${ }^{15}$ Search for Extraterrestrial Intelligence Institute, Mountain View, 189 North Bernardo Ave, Mountain View, CA 94043, USA \\ ${ }^{16}$ NASA Ames Research Center, Moffett Field, Mountain View, CA 94035, USA \\ ${ }^{17}$ Max Planck Institute for Radio Astronomy, Auf dem Hügel 69, 53121, Bonn, Germany \\ ${ }^{18}$ Université Pierre et Marie Curie, 4 Place Jussieu, 75005 Paris, France \\ ${ }^{19}$ Dordt College, 498 4th Ave NE, Sioux Center, IA 51250, USA \\ ${ }^{20}$ Minnesota Institute for Astrophysics, University of Minnesota, 106 Pleasant St. SE Minneapolis, MN 55455, USA \\ Received 2015 July 10; accepted 2015 December 2; published 2016 January 29
}

\begin{abstract}
As gas giant planets and brown dwarfs radiate away the residual heat from their formation, they cool through a spectral type transition from $\mathrm{L}$ to $\mathrm{T}$, which encompasses the dissipation of cloud opacity and the appearance of strong methane absorption. While there are hundreds of known T-type brown dwarfs, the first generation of directly imaged exoplanets were all L type. Recently, Kuzuhara et al. announced the discovery of GJ $504 \mathrm{~b}$, the first T dwarf exoplanet. GJ $504 \mathrm{~b}$ provides a unique opportunity to study the atmosphere of a new type of exoplanet with a $\sim 500 \mathrm{~K}$ temperature that bridges the gap between the first directly imaged planets $(\sim 1000 \mathrm{~K})$ and our own solar system's Jupiter $(\sim 130 \mathrm{~K})$. We observed GJ $504 \mathrm{~b}$ in three narrow $L$-band filters $(3.71,3.88$, and $4.00 \mu \mathrm{m})$, spanning the red end of the broad methane fundamental absorption feature $(3.3 \mu \mathrm{m})$ as part of the LBTI Exozodi Exoplanet Common Hunt (LEECH) exoplanet imaging survey. By comparing our new photometry and literature photometry with a grid of custom model atmospheres, we were able to fit GJ 504 b's unusual spectral energy distribution for the first time. We find that GJ $504 \mathrm{~b}$ is well fit by models with the following parameters: $T_{\text {eff }}=544 \pm 10 \mathrm{~K}, \quad g<600 \mathrm{~m} \mathrm{~s}^{-2}, \quad[\mathrm{M} / \mathrm{H}]=0.60 \pm 0.12, \quad$ cloud opacity parameter of $f_{\text {sed }}=2-5$, $R=0.96 \pm 0.07 R_{\mathrm{Jup}}$, and $\log (L)=-6.13 \pm 0.03 L_{\odot}$, implying a hot start mass of $3-30 M_{\text {jup }}$ for a conservative age range of 0.1-6.5 Gyr. Of particular interest, our model fits suggest that GJ 504 b has a superstellar metallicity. Since planet formation can create objects with nonstellar metallicities, while binary star formation cannot, this result suggests that GJ $504 \mathrm{~b}$ formed like a planet, not like a binary companion.
\end{abstract}

Key words: planets and satellites: atmospheres - planets and satellites: composition - planets and satellites: gaseous planets - stars: individual (GJ 504)

\footnotetext{
* The LBT is an international collaboration among institutions in the United States, Italy, and Germany. LBT Corporation partners are the University of Arizona on behalf of the Arizona university system; Istituto Nazionale di Astrophisica, Italy; LBT Beteiligungsgesellschaft, Germany, representing the Max-Planck Society, the Astrophysical Institute Potsdam, and Heidelberg University; The Ohio State University, and the Research Corporation, on behalf of the University of Notre Dame, University of Minnesota, and University of Virginia.
}

\section{INTRODUCTION}

When brown dwarfs cool below $\sim 1200 \mathrm{~K}$, their atmospheres transition from cloudy to clear, and methane becomes a dominant absorber in their spectral energy distributions (SEDs). Although gas giants were thought to be analogs to brown dwarfs, the first generation of directly imaged exoplanets had cloudy, methane-free atmospheres, even though their temperatures are well below the temperature where field 
brown dwarfs have had their " $\mathrm{L} \rightarrow \mathrm{T}$ " transition (Chauvin et al. 2004; Marois et al. 2008, 2010; Skemer et al. 2011). GJ $504 \mathrm{~b}$, discovered by the SEEDS survey (Strategic Explorations of Exoplanets and Disks with Subaru; Tamura 2009), is the first example of an exoplanet that is cold enough $(\sim 500 \mathrm{~K})$ to be relatively cloud-free and have strong methane absorption features (Janson et al. 2013; Kuzuhara et al. 2013). Another T-dwarf exoplanet was recently discovered around 51 Eri (Macintosh et al. 2015).

At a separation of 2". 5 (43.5 AU) from its G-star host, GJ $504 \mathrm{~b}$ is easily accessible to most high-contrast imaging systems (Kuzuhara et al. 2013). Its $H-K_{s}$ color $(0.63 \pm 0.15)$ is highly discrepant with similar-luminosity field brown dwarfs (approximately -0.2; Dupuy \& Liu 2012). At the same time, it has strong methane absorption at $1.66 \mu \mathrm{m}$, clearly placing it in a different class than other directly imaged exoplanets (Janson et al. 2013). GJ 504 also has supersolar metallicity ([M/ $\mathrm{H}]=0.10-0.28$, although most determinations are toward the lower end of this range; Edvardsson et al. 1993; Mishenina et al. 2004; Valenti \& Fischer 2005; Takeda 2007; Gonzalez et al. 2010; Maldonado et al. 2012; Ramírez et al. 2013). It is therefore relatively likely to have a gas giant planet (Fischer \& Valenti 2005).

The age of GJ 504 A, and thus the mass and planetary status of GJ 504 b, is uncertain. Kuzuhara et al. (2013) find consistency among multiple age indicators, such as X-ray activity, rotation rate, chromospheric activity, and $\mathrm{H}-\mathrm{R}$ diagram location, indicating an age for GJ 504 A of $0.1-0.5 \mathrm{Gyr}$. However, a reanalysis of GJ 504 A's stellar properties by Fuhrmann \& Chini (2015) suggests that the star lies above the main sequence on an H-R diagram with a corresponding age of 4.5 Gyr. Fuhrmann \& Chini (2015) argue that the rapid rotation and other signs of youth arise because a massive planet has fallen into the star, carrying its orbital angular momentum with it. This leaves the presence of strong lithium absorption (Kuzuhara et al. 2013) unexplained (Soderblom 2010). Some of the lithium could have been replenished by the planet (Carlberg et al. 2012), but an usually massive planet would be required. Since there is no consensus on the age of the system, we consider both estimates in the following discussion; the younger age range implies a planet mass of $\sim 3-9 M_{\text {Jup }}$, while the older one would suggest a mass of $\sim 30 M_{\text {Jup }}$.

The LBTI Exozodi Exoplanet Common Hunt (LEECH) is a 100-night survey with the Large Binocular Telescope (LBT) to search for and characterize exoplanets in the mid-infrared (Skemer et al. 2014a; Maire et al. 2015). In this work, we confirm the detection of GJ 504 b and obtain photometry of the first $\mathrm{T}$-dwarf exoplanet in three narrow $L$-band filters (3.71, 3.88 , and $4.00 \mu \mathrm{m})$. For $\mathrm{T}$ dwarfs, $L$-band photometry can probe the broad methane fundamental absorption feature (centered at $3.3 \mu \mathrm{m}$ ) and put strong constraints on the luminosity of the planet, which peaks at $\sim 4 \mu \mathrm{m}$. The overall benefit of this additional photometry is to improve our ability to constrain GJ 504 b's bulk properties with atmospheric modeling. In Section 2, we present our observations and reductions, which comprise some of the deepest images taken from the ground at these wavelengths. In Section 3, we present our new photometry and adjust the literature photometry onto a common photometric system. In Section 4, we fit the photometry with a grid of models and discuss the physical nature of GJ 504 b. Finally, we present our conclusions in Section 5 .
Table 1

Filter Properties

\begin{tabular}{lccc}
\hline \hline Filter & $\lambda_{\text {eff }}(\mu \mathrm{m})$ & FWHM $(\mu \mathrm{m})$ & Zero-point Flux $(\mathrm{Jy})$ \\
\hline$L_{\mathrm{NB} 6}$ & 3.71 & 0.19 & 257 \\
$L_{\mathrm{NB} 7}$ & 3.88 & 0.23 & 239 \\
$L_{\mathrm{NB} 8}$ & 4.00 & 0.06 & 224 \\
\hline
\end{tabular}

Note. Manufacturer's curves for $L_{\mathrm{NB} 1}-L_{\mathrm{NB} 7}$ were previously used and tabulated in Skemer et al. (2014b). $L_{\mathrm{NB} 8}$ is an additional filter at longer wavelength. Here we include the filters' cryogenic shifts based on an on-sky wavelength calibration of the LMIRcam grism (Skrutskie et al. 2014).

\section{OBSERVATIONS AND REDUCTIONS}

We observed GJ 504 on UT 2013 April 21 and UT 2014 March 11-13 with the Large Binocular Telescope Interferometer (LBTI; Hinz et al. 2012) and its $1-5 \mu \mathrm{m}$ imager, $L / M$ Infrared Camera (LMIRcam; Skrutskie et al. 2010; Leisenring et al. 2012). The LBT has twin deformable secondary adaptive optics (AO) systems (Esposito et al. 2011; Bailey et al. 2014), which provide excellent sensitivity in the thermal infrared $(\gtrsim 2 \mu \mathrm{m})$ compared to traditional AO systems (LloydHart 2000). The diffraction-limited beams from the AO systems are fed into LBTI, which can overlap or separate the two images on LMIRcam. For contrast-limited observations, such as the LEECH planet search (Skemer et al. 2014a), we typically separate the beams to allow independent and redundant observations of the inner, speckle-noise-limited regime. For sensitivity-limited observations, we overlap the beams (incoherently) so that the faint astronomical source can be extracted from a single bright sky background aperture, rather than two. GJ 504 b, at a separation of $\sim 2$ ". 5 , falls into the latter category, where sensitivity is a greater priority than contrast. For the UT 2013 April 21 observations, the LBT's right-side adaptive optics system was unavailable, so we acquired data using just the left side of the telescope. For the UT 2014 March 11-13 observations, both sides of the telescope were operable, and we overlapped the two images of GJ 504 b to increase our sensitivity.

We observed GJ 504 in narrowband filters: $L_{\mathrm{NB} 6}$ (3.61-3.80 $\mu \mathrm{m}), \quad L_{\mathrm{NB} 7} \quad(3.76-3.99 \mu \mathrm{m}), \quad$ and $L_{\mathrm{NB} 8}$ $(3.97-4.03 \mu \mathrm{m})$. Basic properties for these filters are tabulated in Table 1, and transmission profiles are shown in Figure 1. The weather was photometric on the nights we obtained $L_{\mathrm{NB} 6}$ (UT 2014 March 12) and $L_{\mathrm{NB} 7}$ (UT 2013 April 21) data. The first night of $L_{\mathrm{NB} 8}$ observations (UT 2014 March 11) was nonphotometric, which prompted us to repeat this filter on UT 2014 March 13. UT 2014 March 13 had patchy clouds away from the telescope, which cleared early in the observations. Integration times were chosen to be long enough that the offstar data were sky background noise limited, rather than read noise limited. This choice meant that the star was saturated in the frames used to detect GJ 504 b. Additional frames with shorter integration times were used to measure the brightness of the host star. A summary of our observations is presented in Table 2 .

We reduced the data using a custom LMIRcam pipeline developed at MPIA (Bonnefoy et al. 2014). The pipeline (1) replaces bad detector pixels with the median of their adjacent neighbors, based on a table of outlier pixels cataloged from offsky calibration frames; (2) removes the detector bias and background sky/telescope emission by subtracting the median 


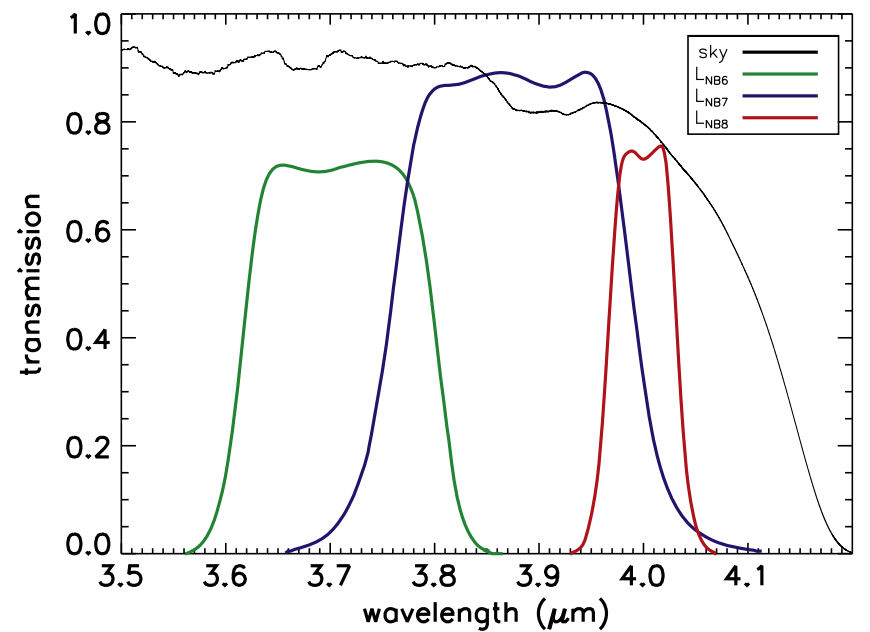

Figure 1. Transmission profiles for the filters used in this paper and a telluric transmission profile for 1.0 airmasses and $4.3 \mathrm{~mm}$ precipitable water vapor from Gemini (http://www.gemini.edu/sciops/telescopes-and-sites/observingcondition-constraints/ir-transmission-spectra).

of images from chronologically neighboring nod subsequences; (3) determines the subpixel centroid of the star point-spread function (PSF) in each image by fitting a Gaussian, masking the inner saturated pixels, and shifts and crops the image to a common, aligned frame; (4) flags images with peak star fluxes below a specified threshold and images with abnormal background levels to exclude data contaminated by clouds and poor seeing; (5) removes residual detector bias from columns and rows, based on overscan pixels; and (6) forms the cube of reduced, photometric-quality images, for inspection and PSF subtraction. Angular differential imaging (ADI) and principal component analysis (PCA) PSF subtraction is carried out with a separate program described later in this section.

Figure 2 shows the result of aligning, de-rotating, and coadding all of the photometric quality images acquired of GJ $504 \mathrm{~b}$ in the $L_{\mathrm{NB} 7}(3.95 \mu \mathrm{m})$ filter - a composite with an effective integration time of $3121 \mathrm{~s}$. Owing to the relatively wide angular separation of GJ 504 b, the planet is visible without subtracting the star, in the northwest corner of the coadded image. The image flux scale is given in units of the star's peak intensity, determined from the short-exposure (unsaturated PSF) image sequence.

To reduce starlight contamination in the planet signal, we subtract an estimate of the star's PSF from each image using standard high-contrast imaging techniques. The data were taken in ADI mode (Marois et al. 2006), where the instrument does not rotate (LBTI is on a nonrotating mount), so that its diffractive pattern and static aberrations stay fixed, while the sky image rotates with parallactic angle. We then reduced the data with a PCA high-contrast algorithm (Amara \& Quanz 2012; Soummer et al. 2012; Fergus et al. 2014), using a custom implementation that closely follows Soummer et al. (2012). At each separation, we optimize the number of principle components that are used in the subtraction $\left(N_{\mathrm{PC}}\right)$ by inserting 12 artificial planets at different azimuth angles and measuring their signal-to-noise ratios as a function of $N_{\mathrm{PC}}$. We also optimize the minimum parallactic rotation gap (parameterized as $N_{\text {FHWM }}$ ) between the image being fit and the library of images used to do the fit (Lafrenière et al. 2007). Optimal values of $N_{\text {PC }}$ vary from $\sim 15$ to 30 , and optimal values of
$N_{\text {FHWM }}$ vary from $\sim 0.5$ to 1.0 . Final reduced images are shown in Figure 3. We detect GJ 504 b at signal-to-noise ratios of 5.9, 5.3, and 5.9 in $L_{\mathrm{NB} 6}, L_{\mathrm{NB} 7}$, and $L_{\mathrm{NB} 8}$, respectively (as described below).

Relative photometry between GJ $504 \mathrm{~A}$ and b is measured using the forward modeling approach described in Soummer et al. (2012), which uses an image of the star at the position of the planet to calibrate the planet self-subtraction that is common to high-contrast image processing techniques (Lafrenière et al. 2007). We optimize the source model over an annular sector centered on the planet and spanning $30^{\circ}$ in position angle, conservatively fixing $N_{\text {FHWM }}=1.0$, as we find that values less than this increase self-subtraction. We measure the error on our relative photometry by inserting artificial planets at the same separation as GJ 504 b, but at different position angles, and repeating the forward modeling. The standard deviation of our measurements of the artificial planets is adopted as our formal relative photometry uncertainty and our detection signal-to-noise ratio. Relative photometry between GJ $504 \mathrm{~A}$ and $\mathrm{b}$ is reported in Table 3. Note that the two nights of $L_{\mathrm{NB} 8}$ observations were combined in this analysis, after confirming that they produced similar photometry, within $1 \sigma$ errors.

\section{PHOTOMETRY}

To convert contrast measurements (relative photometry) to apparent magnitudes, we adopt and calculate photometry for GJ 504 A. For $J, H$, and $K_{s}$, we adopt apparent photometry from Kidger \& Martín-Luis (2003), converted to Two Micron All Sky Survey photometry $\quad(J=4.13 \pm 0.01$, $\left.H=3.88 \pm 0.01, K_{s}=3.81 \pm 0.01\right)$ using the methodology and Vega spectrum of Rieke et al. (2008). For all other filters where GJ 504 b has been observed, we calculate GJ 504 A's apparent photometry by fitting a model stellar atmosphere (Castelli \& Kurucz 2004) with parameters that best match GJ 504's temperature, gravity, and metallicity measurements (Valenti \& Fischer 2005). In our fit, we use the previously quoted $J H K_{s}$ photometry, as well as WISE (Cutri et al. 2013) $\mathrm{W} 3(3.831 \pm 0.015)$ and W4 $(3.757 \pm 0.022)$ photometry (the WISE W1 and W2 filters were not used because they were flagged as saturated). Our fit to these five data points with a model atmosphere produced a reduced $\chi^{2}$ of 0.70 . We estimate GJ 504 A's apparent magnitude to be 3.87 in $\mathrm{CH}_{4} \mathrm{~s}, 3.85$ in $\mathrm{CH}_{4} 1$, and 3.82 in $L^{\prime}, L_{\mathrm{NB} 6}, L_{\mathrm{NB} 7}$, and $L_{\mathrm{NB} 8} \cdot{ }^{20}$ This modeldriven approach leads to a $1 \sigma-2 \sigma$ inconsistency between our $\mathrm{CH}_{4}$ photometry and our $\mathrm{H}$-band photometry (the two $\mathrm{CH}_{4}$ bands span $H$ band and should not be brighter than the $H$ photometry), which we would like to correct in order to avoid propagating erroneous color information into GJ 504 b's photometry (incorrect colors could affect our derived model atmosphere parameters, whereas an incorrect overall luminosity will only affect radius). Therefore, we adjust our estimated $\mathrm{CH}_{4}$ photometry to be fainter by $0.02 \mathrm{mag}$ to be consistent with the broad $H$-band value. Similarly, we adjust the $L$ photometry to be brighter by $0.02 \mathrm{mag}$ to be consistent with the $K_{s}$ photometry. Since inconsistencies in our photometric estimates appear to be at the $\sim 0.02 \mathrm{mag}$ level, we adopt $0.02 \mathrm{mag}$ uncertainties for all GJ 504 A photometry. These

\footnotetext{
${ }^{20}$ Our $L$ estimates (for all four filters) are 0.12 mag different from the $L^{\prime}$ estimate of Kuzuhara et al. (2013), who used a photometric measurement of GJ 504 A with a large (0.09 mag) uncertainty. Our model-based estimate is more precise and is consistent with the $J H K$ photometry.
} 
Table 2

Observations

\begin{tabular}{|c|c|c|c|c|c|}
\hline Date & Filter & Aperture & $\begin{array}{c}\text { Frame Time (Sat/Unsat) } \\
\text { (s) }\end{array}$ & $\begin{array}{l}\text { Int Time } \\
\text { (minutes) }\end{array}$ & Conditions \\
\hline UT 2013 Apr 21 & $L_{\mathrm{NB} 7}$ & $8.4 \mathrm{~m}$ & $0.524 / 0.058$ & 55 & photometric, $0 . " 9$ seeing \\
\hline UT 2014 Mar 11 & $L_{\mathrm{NB} 8}$ & $2 \times 8.4 \mathrm{~m}$ & $0.990 / 0.087$ & 30 & patchy clouds, 1 ". 4 seeing \\
\hline UT 2014 Mar 12 & $L_{\mathrm{NB} 6}$ & $2 \times 8.4 \mathrm{~m}$ & $0.291 / 0.029$ & 101 & photometric, $0 . " 9$ seeing \\
\hline UT 2014 Mar 13 & $L_{\mathrm{NB} 8}$ & $2 \times 8.4 \mathrm{~m}$ & $0.873 / 0.087$ & 44 & patchy clouds and then clear, 1 ". 0 seeing \\
\hline
\end{tabular}

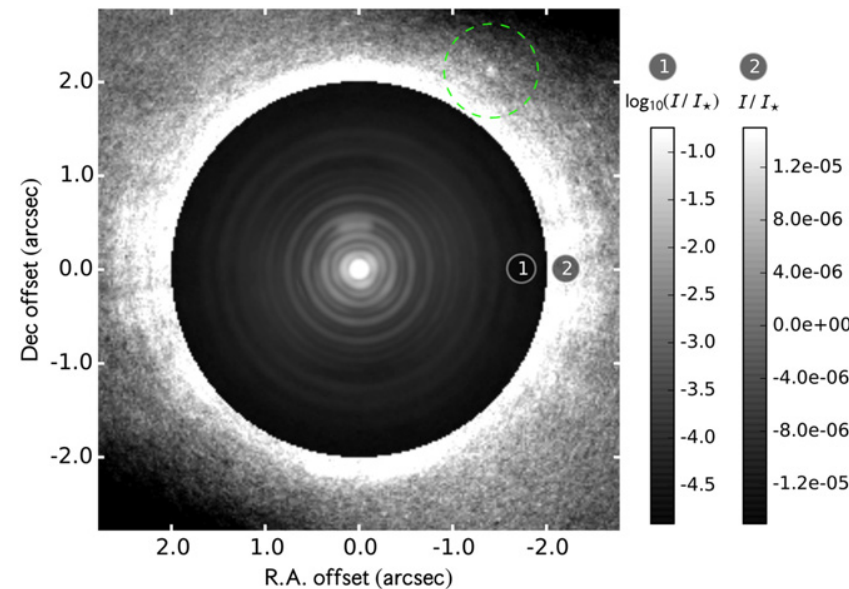

Figure 2. De-rotated co-add of all photometric-quality GJ 504 images taken in the $L_{\mathrm{NB} 7}(3.88 \mu \mathrm{m})$ filter. The planet, GJ $504 \mathrm{~b}$, is the circled point source to the northwest of the star, at separation $\sim 2$ ". 5 . Owing to the large dynamic range of structure in the image, two regions are displayed with different flux scales: (1) within $2^{\prime \prime}$ of the star, the Airy rings are displayed with a logarithmic stretch; the outer region (2) that includes the planet is displayed with a linear stretch centered at zero. The corresponding grayscale bars on the right-hand side indicate the flux in units normalized to the peak of the unsaturated star's PSF.

inconsistencies are independent of our exact choice of stellar parameters and are more likely the result of propagated photometric errors or stellar variability. Our adopted GJ 504 A photometry and the resulting GJ $504 \mathrm{~b}$ photometry are summarized in Table 3. For our atmosphere modeling, we convert to absolute magnitudes assuming a distance of $17.56 \pm 0.08 \mathrm{pc}$ (van Leeuwen 2007).

\section{DISCUSSION: GJ 504 b's UNUSUAL APPEARANCE}

With the first discoveries of directly imaged planets, it was obvious that something about planetary atmospheres made them different from the atmospheres of similar-temperature field brown dwarfs (Chauvin et al. 2004; Marois et al. 2008). In near-infrared color-magnitude diagrams (see Figure 4), the HR 8799 planets fall on what appears to be an extension of the L-dwarf sequence. The implication is that these young planets have retained their dusty, methane-poor atmospheres at lower luminosities than old field brown dwarfs, which transition to methane-rich, cloud-free $\mathrm{T}$ dwarfs below $M_{H} \sim 14$ mag. Surveys have now found young, dusty brown dwarfs that are more analogous to the HR 8799 planets (e.g., Faherty et al. 2013; Liu et al. 2013; Gauza et al. 2015), and there are theoretical justifications for why, in addition to effective temperature, an object's mass/surface gravity affects the clouds and chemistry of its photosphere (Marley et al. 2012; Zahnle \& Marley 2014).

Also plotted in Figure 4 is GJ 504 b, which is much less luminous than previously discovered exoplanets. In the $J-H$ versus $J$ and $K_{s}-L^{\prime}$ versus $K_{s}$ diagrams, GJ 504 b falls right on the late T-dwarf sequence. However, in the $H-K_{s}$ versus $H$ diagram, GJ $504 \mathrm{~b}$ is much redder than the field $\mathrm{T}$ dwarfs. There is one other object near GJ $504 \mathrm{~b}$ in the color-magnitude diagrams: GJ $758 \mathrm{~B}$, a $\sim 30-40 M_{\text {Jup }}$ companion to a $\sim 6 \mathrm{Gyr}$ Sun-like star (Mamajek \& Hillenbrand 2008; Thalmann et al. 2009; Janson et al. 2011).

Clearly, there is something about GJ $504 \mathrm{~b}$ and GJ 758 B that make them different from other objects with similar luminosities. For the HR 8799 planets, youth and low surface gravity are responsible for their unusual appearances (Marois et al. 2008). While GJ 504 b's age is uncertain and GJ 758 B is clearly old, their position in Figure 4 unambiguously demonstrates that at least one of their physical properties is unusual.

For GJ 504 b, Kuzuhara et al. (2013) and Janson et al. (2013) suggest that gravity or metallicity could be driving the planet's unusual near-infrared colors. In the rest of this section, we attempt to model GJ 504 b's atmosphere and directly constrain these properties.

\subsection{Atmosphere Models}

We attempt to fit the complete SED of GJ 504 b using the photometry described in Section 3. We use models similar to those described in Morley et al. (2012, 2014), which include opacities for $\mathrm{T} / \mathrm{Y}$-dwarf condensates. The methane line lists have been updated using Yurchenko et al. (2014), and the alkali line lists have been updated to use the results from Allard et al. (2005). Chemical equilibrium grids based on previous thermochemical models (Lodders 1999, 2002; Lodders \& Fegley 2002, 2006; Visscher et al. 2006, 2010; Visscher 2012; Moses et al. 2013) have been revised and extended to include higher metallicities. These updates will be described in detail in a set of upcoming papers that focus on the new model grid. In addition to temperature, our model grid parameterizes surface gravity, metallicity, and cloud thickness. ${ }^{21} \mathrm{We}$ allow radius to be a free parameter so that atmospheric properties rather than luminosity drive the fit. Our model grid contains 480 models, comprising temperatures of $450,475,500,525,550,575,600$, and $625 \mathrm{~K}$, surface gravities of $30,100,300$, and $1000 \mathrm{~m} \mathrm{~s}^{-2}$, metallicities of $[\mathrm{M} / \mathrm{H}]=0,0.5$, and 1.0 , and cloud thicknesses of $f_{\text {sed }}=1,2,3,5$, and cloud-free. The parameters that we choose to vary are among the most fundamental to the bulk appearance of planetary atmospheres. However, we cannot rule out that additional parameters, such as nonequilibrium $\mathrm{NH}_{3}$ chemistry (Zahnle \& Marley 2014), might play an important role as well. We also note that systematic differences between model families can account for substantially disparate

\footnotetext{
21 Cloud opacity is parameterized as a particle sedimentation efficiency, labeled $f_{\text {sed }}$, as described by Ackerman \& Marley (2001). Lower $f_{\text {sed }}$ numbers correspond to larger cloud opacities.
} 

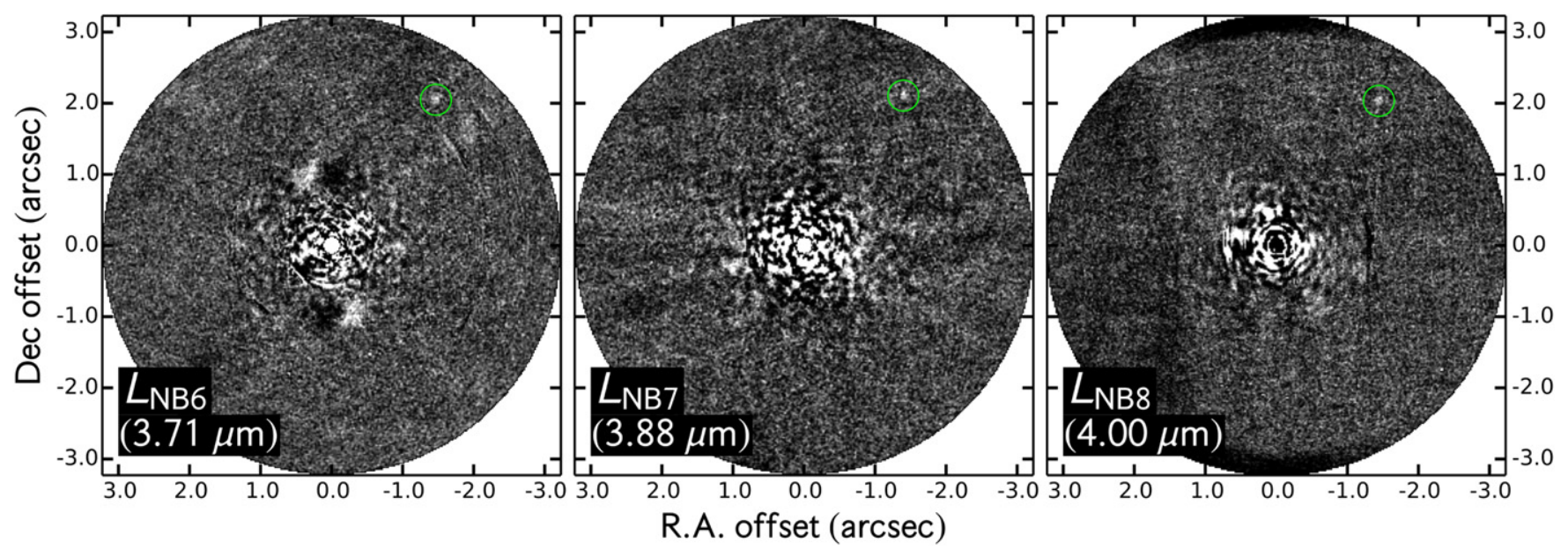

Figure 3. Final starlight-subtracted images of data taken in the $L_{\mathrm{NB} 6}, L_{\mathrm{NB} 7}$, and $L_{\mathrm{NB} 8}$ filters.

Table 3

GJ 504 Photometry (Apparent Magnitudes)

\begin{tabular}{|c|c|c|c|c|c|c|c|c|c|}
\hline Object & $\begin{array}{c}J \\
(1.25 \mu \mathrm{m})\end{array}$ & $\begin{array}{c}H \\
(1.63 \mu \mathrm{m})\end{array}$ & $\begin{array}{c}\mathrm{CH}_{4} \mathrm{~S} \\
(1.56 \mu \mathrm{m})\end{array}$ & $\begin{array}{c}\mathrm{CH}_{4} \mathrm{l} \\
(1.71 \mu \mathrm{m})\end{array}$ & $\begin{array}{c}K s \\
(2.15 \mu \mathrm{m})\end{array}$ & $\begin{array}{c}L^{\prime} \\
(3.78 \mu \mathrm{m})\end{array}$ & $\begin{array}{c}L_{\mathrm{NB} 6} \\
(3.71 \mu \mathrm{m})\end{array}$ & $\begin{array}{c}L_{\mathrm{NB} 7} \\
(3.88 \mu \mathrm{m})\end{array}$ & $\begin{array}{c}L_{\mathrm{NB} 8} \\
(4.00 \mu \mathrm{m})\end{array}$ \\
\hline GJ $504 \mathrm{~A}$ & 4 & 02 & $3.89 \pm$ & 3.87 & $3.81=$ & 3.80 & 3.8 & 3.80 & $3.80=$ \\
\hline $\begin{array}{l}\text { GJ } \\
504 \\
\text { b-A }\end{array}$ & $15.65 \pm$ & $16.13=$ & $15.71 \pm$ & $>$ & $15.57=$ & $12.90=$ & 13.79 & $12.67 \pm 0.19$ & 12.05 \\
\hline GJ 504 b & $19.78 \pm 0.10$ & $20.01 \pm 0.10$ & $19.60 \pm 0.12$ & $>20.64(3 \sigma)$ & $19.38 \pm 0.11$ & $16.70 \pm 0.17$ & $17.59 \pm 0.17$ & $16.47 \pm 0.19$ & $15.85 \pm 0.17$ \\
\hline
\end{tabular}

Note. The GJ 504 A photometry has been recalculated, as described in Section 3. Relative photometry is from Janson et al. (2013) for $J$, $H$, CH4s, CH4l, and $K_{s}$, Kuzuhara et al. (2013) for $L^{\prime}$, and this work for $L_{\mathrm{NB} 6}, L_{\mathrm{NB} 7}$, and $L_{\mathrm{NB} 8}$.

parameter estimates (Patience et al 2012). With these caveats in mind, our best-fit model is $T=550 \mathrm{~K},[\mathrm{M} / \mathrm{H}]=0.5$, $R=0.94 R_{\mathrm{Jup}}, \quad g=100 \mathrm{~m} \mathrm{~s}^{-2}, \quad f_{\mathrm{sed}}=3, \quad$ and $\quad \log$ $(L)=-6.13 L_{\odot} \quad\left(\right.$ see Figure 5). The reduced $\chi^{2}$ (counting only radius scaling as a free parameter) is 0.98 with 7 degrees of freedom. None of the other models provide a fit with a relative probability higher than $1 \%$, based on the Bayesian probability: $P\left(\operatorname{model}_{1} / \operatorname{model}_{2}\right)=e^{\left(\chi_{\text {model }}^{2}-\chi_{\text {model } 1}^{2}\right)} / 2$ for Gaussian photometric errors and a uniform model prior. While this analysis demonstrates that there is at least one self-consistent model that adequately fits all of the data, our current grid is too sparse to sample the error distribution of each parameter. Without sampling the error distribution, our best-fit model may not be at the peak of the global probability distribution. Thus, in Section 4.2, we interpolate between the models to form a denser grid, which we use to adopt estimates for each parameter.

To lend some intuition to the effects of varying individual parameters, Figure 6 shows the best-fit model in four panels, which individually vary temperature, surface gravity, metallicity, and cloud properties. As with our best fit, all models are scaled by radius. In this scheme (which is partially driven by the size of the error bars and the radius fit), temperature is primarily constrained by the $L^{\prime}$ - and narrow $L$-band photometry. Gravity is constrained by the photometry in the $J$ and $L$ bands. Metallicity is constrained by $J, K_{s}$, and $L$. Cloud properties are also constrained by $J, K_{s}$, and $L$. However, for metallicity, $K_{s}$ and $L$ move in opposite directions, while for cloud properties, they move in the same direction. No parameter is fully degenerate with a combination of other parameters, and all four parameters (plus radius) were necessary to obtain an adequate best fit. The $L$-band photometry, in particular, was critical for resolving degeneracies between temperature and the other parameters.

\subsection{Interpolated Atmosphere Models}

While our model grid is able to produce a plausible fit to the available GJ 504 b data, it is too sparsely spaced to sample the error distribution of the model parameters. We cannot easily produce a much larger grid of models, so instead, we interpolate between the models using quad-linear interpolation (linear with temperature, metallicity, and cloud properties, logarithmic with surface gravity). We assign probabilities to each model using the Bayesian posterior, $P \propto e^{-\chi^{2} / 2}$, with Bayesian priors set by grid spacing. We adopt uniform priors for temperature, $f_{\text {sed }}$, and $[\mathrm{M} / \mathrm{H}]$ over their full model-allowed range. For gravity, we adopt uniform priors above a minimum surface gravity that is set by the radius of the planet and the minimum planet mass $\left(3 M_{\text {jup }}\right)$ derived by Kuzuhara et al. (2013). For radius we adopt a uniform prior between 0.9 and $1.3 R_{\mathrm{Jup}}$, the plausible radius range for GJ $504 \mathrm{~b}$, varying mass, core mass, initial entropy, and metallicity (Fortney et al. 2007, 2008). Marginal probabilities are shown in Figure 7. Gaussian fits to the marginalized probability distributions give the following marginalized parameter distributions for GJ 504 b: $\quad T_{\text {eff }}=544 \pm 10 \mathrm{~K}, \quad[\mathrm{M} / \mathrm{H}]=0.60 \pm 0.12$, $R=0.96 \pm 0.07 R_{\odot}, \log (L)=-6.13 \pm 0.03 L_{\odot}$. For cloudiness and gravity, whose distributions do not resemble Gaussians, we adopt $f_{\text {sed }}$ (cloudiness) $=2-5$ and $g<600 \mathrm{~m} \mathrm{~s}^{-2}$. Parallax uncertainty has a negligible effect on the radius and luminosity errors. Two-dimensional probability 

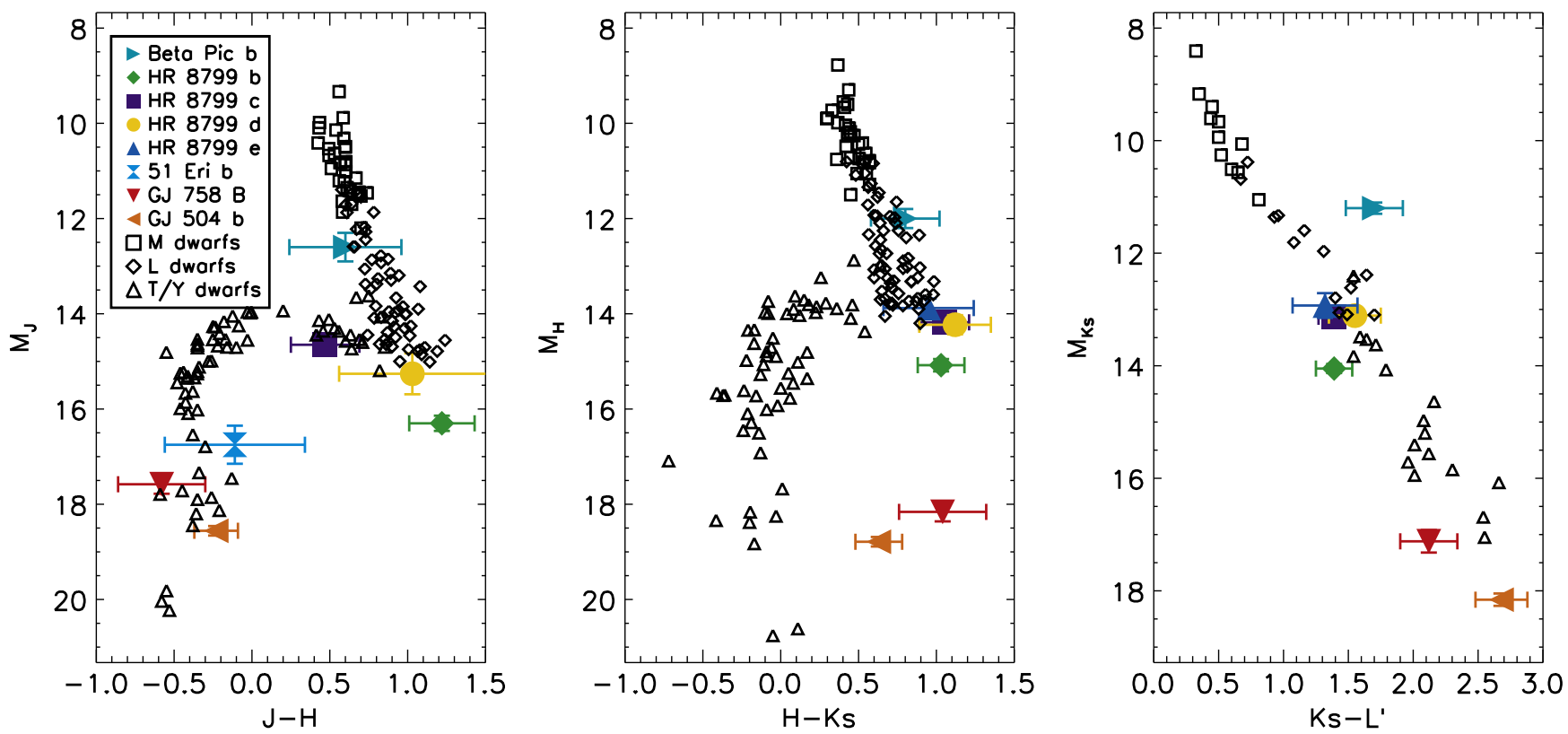

Figure 4. Color-magnitude diagrams showing the field $\mathrm{M} \rightarrow \mathrm{L} \rightarrow \mathrm{T}$ brown dwarf sequence and directly imaged exoplanets. In the middle diagram $\left(H-K_{s}\right.$ vs. $\left.H\right)$, GJ $504 \mathrm{~b}$ is highly discrepant with similar-magnitude field brown dwarfs. The directly imaged brown dwarf, GJ $758 \mathrm{~B}$, has a similar appearance. Photometry is compiled from Bonnefoy et al. (2011, 2013), Marois et al. (2008, 2010), Skemer et al. (2012), Kuzuhara et al. (2013), Janson et al. (2011, 2013), Dupuy \& Liu (2012), and Macintosh et al. (2015), with some adjustments described in Section 3 (note that GJ 758 B's $K$ photometry is $K_{c}$, not $K_{s}$ ). The brown dwarf photometry has been selected to have errors smaller than $0.1 \mathrm{mag}$ in each filter and uses $K_{\mathrm{MKO}}$ instead of $K_{s}$, which is more complete for later spectral types and produces qualitatively similar results.

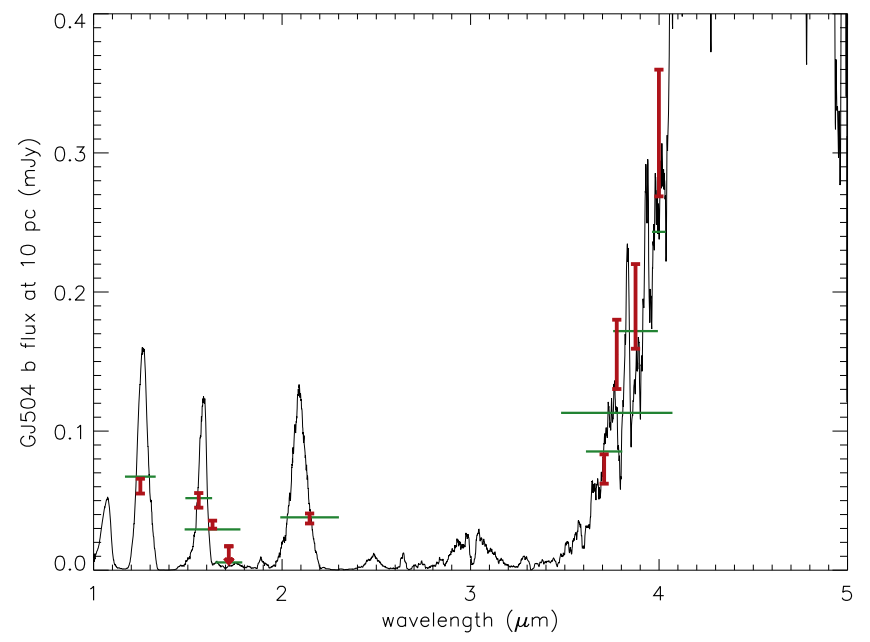

Figure 5. Photometry of GJ $504 \mathrm{~b}$ with a best-fit model photosphere. The red error bars and $1.7 \mu \mathrm{m}$ upper limit are photometry tabulated in Table 3. The green horizontal bars are model photometry, with the width of the bars denoting the filter bandpass. The black curve is the best fit from a grid of atmosphere models that vary temperature, surface gravity, metallicity, and cloud type, with radius scaling as a free parameter. The reduced $\chi^{2}$ (counting only radius scaling as a free parameter) is 0.98 .

contours, for each pair of model parameters, are shown in Figure 8. Correlations are evident between parameters.

Interpolation carries an intrinsic risk that the spectra change in nonlinear ways, which can vary by bandpass. For our particular model grid and photometry, the best example of this behavior is seen in the metallicity plot of Figure 6 . The $J$-band photometry changes more quickly between $[\mathrm{M} / \mathrm{H}]=0.5$ and 1.0 than between $[\mathrm{M} / \mathrm{H}]=0.0$ and 0.5 . At the same time, many of the other model photometry points move linearly with $[\mathrm{M} / \mathrm{H}]$. To test how this might affect our final marginalized probability distributions, we repeated our analysis interpolating exponentially $\left(10^{[\mathrm{M} / \mathrm{H}]}\right)$ in the $J$ band and linearly in the other bands. The net result is a shift in the probability distribution of $3 \mathrm{~K}$ for $T_{\text {eff }}, 0.02$ for $[\mathrm{M} / \mathrm{H}], 0.02 R_{\text {Jup }}$ for radius, and negligible changes for the other parameters. In all cases, this shift is much smaller than our derived error bars.

\subsection{The Physical Properties of GJ $504 b$}

Our model fitting constrains several physical properties of the planet that can help us understand its formation and evolution.

\subsubsection{Temperature and Radius}

We estimate GJ 504 b's temperature to be $T_{\text {eff }}=544 \pm 10 \mathrm{~K}$ and its radius to be $R=0.96 \pm 0.07 R_{\text {Jup }}$. To first order, these parameters are highly correlated (for a blackbody, $T_{\text {eff }} \propto R^{-0.5}$ ). At this point, ultracool atmosphere models are not calibrated to the point that the radius estimate could tell us much about the planet's internal structure.

\subsubsection{Luminosity and Mass}

Our derived bolometric luminosity of $\log (L)=$ $-6.13 \pm 0.03 L_{\odot}$ is only somewhat lower than the Kuzuhara et al. (2013) estimate of $-6.09_{-0.08}^{+0.06}$. Kuzuhara et al. (2013) estimate bolometric luminosity by averaging the Baraffe et al. (2003) model values that correspond with their individual photometric points. The anomalously bright $K_{s}$ photometry, as they note, biases this value toward higher luminosities. Because our atmosphere models are able to fit all of the photometry simultaneously, they provide a more reliable indicator of bolometric luminosity.

Our revised bolometric luminosity motivates an updated estimate of GJ 504 b's mass. Using the Kuzuhara et al. 


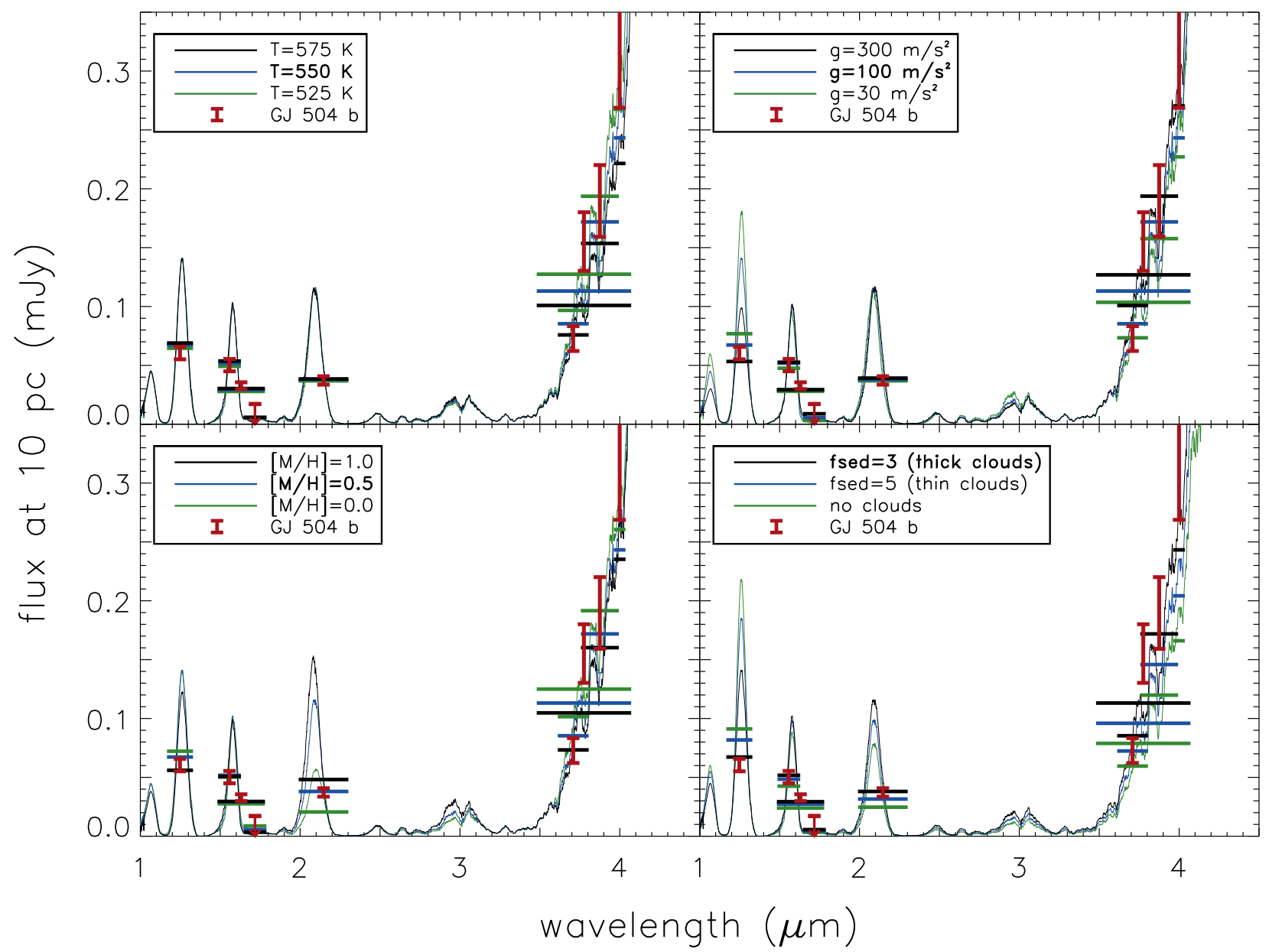

Figure 6. Effects of varying model parameters. For temperature, surface gravity, metallicity, and cloud type, we show the best-fit model (bold in each legend) along with additional models that vary that parameter. All models have radius scaling as a free parameter. In this scheme (which is partially driven by the size of the error bars and the radius fit), temperature primarily affects 3-4 $\mu \mathrm{m}$ photometry. Gravity affects the $J$ and the 3-4 $\mu \mathrm{m}$ photometry. Metallicity affects $J, K s$, and the 3-4 $\mu \mathrm{m}$ photometry. Cloud properties also affect $J, K_{s}$, and the 3-4 $\mu \mathrm{m}$ photometry. However, for metallicity, $K_{s}$ and the 3-4 $\mu \mathrm{m}$ move in opposite directions, while for cloud properties, they move in the same direction. Thus, the parameters are not degenerate.

(2013) age (0.1-0.51 Gyr) and Baraffe et al. (2003) models, we find that GJ $504 \mathrm{~b}$ has a mass of 3-8 $M_{\text {jup }}$. Using the Fuhrmann \& Chini (2015) age (3-6.5 Gyr) and Baraffe et al. (2003) models, we find that GJ $504 \mathrm{~b}$ has a mass of $19-30 M_{\text {jup }}$.

\subsubsection{Metallicity}

With a metallicity of $[\mathrm{M} / \mathrm{H}]=0.60 \pm 0.12$, GJ $504 \mathrm{~b}$ appears to be metal-rich, even when compared to its slightly metal-rich host star $(\mathrm{M} / \mathrm{H}=0.10-0.28$; Edvardsson et al. 1993; Mishenina et al. 2004; Valenti \& Fischer 2005; Takeda 2007; Gonzalez et al. 2010; Maldonado et al. 2012; Ramírez et al. 2013). Based on Figure 6, the high metallicity has a large effect on GJ 504 b's bright $K_{s}$ photometry, which explains the object's unusual placement on the $H-K_{s}$ versus $H$ color-magnitude diagram in Figure 4.

The ability to measure the metallicity of individual planets, whether by broadband photometry or by line-resolving spectroscopy, is hugely important for understanding the formation and evolution of extrasolar planets. Core-accretion theory predicts that gas giant exoplanets should have higher metallicities than their host stars owing to the infall of planetessimals (Pollack et al. 1986; Podolak et al. 1988). In some circumstances, gravitational instability can also produce planets with metallicities that vary from their host stars (Boley
\& Durisen 2010), and indeed, higher metallicities may be favored (Nayakshin 2015). While planet formation can create an object whose metallicity differs from its host star, binary star formation will not generally create objects with vastly different metallicities (Desidera et al. 2004, 2006). Therefore, within the confines of our atmospheric modeling, it appears that GJ $504 \mathrm{~b}$ formed like a planet, not like a binary star.

$$
\text { 4.3.4. Clouds }
$$

GJ $504 \mathrm{~b}$ appears to have some cloud opacity. Although dense silicate clouds are not seen in objects as cold as GJ 504 b, other condensates are predicted to affect the SEDs of cool atmospheres (Morley et al. 2012). Low-surface-gravity objects can support clouds at lower atmospheric pressures than highsurface-gravity objects (Marley et al. 2012), although it remains to be seen whether GJ 504 b's cloud properties are inconsistent with the cloud properties of more massive field brown dwarfs.

\subsubsection{Surface Gravity and Age}

Our analysis shows a preference for low surface gravities, constrained at the low end by evolutionary models, rather than atmosphere models (see our Bayesian prior in Section 4.2). A low surface gravity suggests that GJ 504 b is a young, low- 

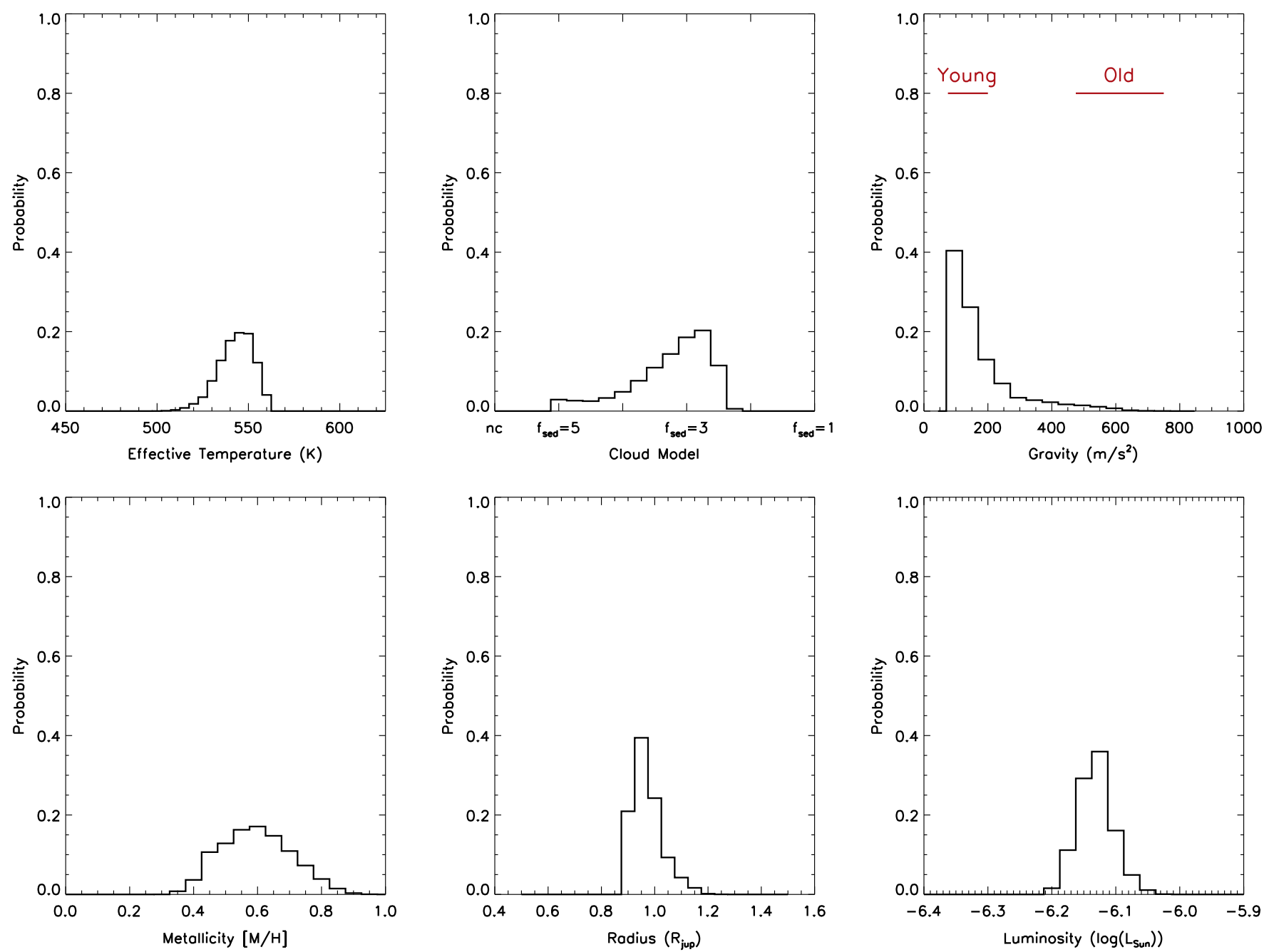

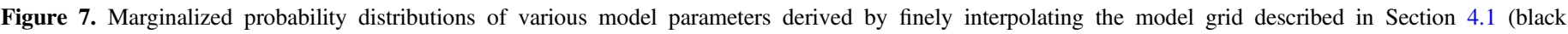

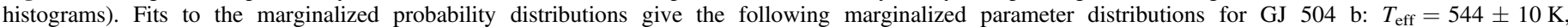

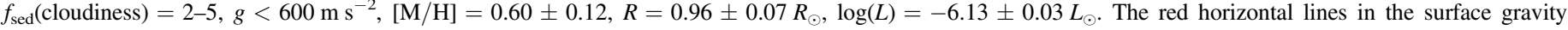

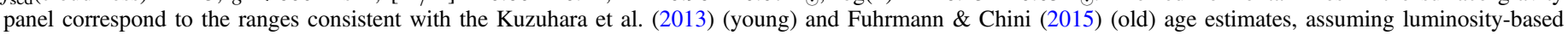
masses from the Baraffe et al. (2003) evolutionary models and $R=1.0 R_{\odot}$.

mass planet (Kuzuhara et al. 2013) rather than an older and more massive brown dwarf (Fuhrmann \& Chini 2015); however, our posterior distribution does not completely rule out the latter.

If the age from Fuhrmann \& Chini (2015) is adopted, the correlations in Figure 8 show that the planet's cloud thickness is decreased and its metallicity is increased. To quantify this, we recalculate the posterior probabilities with the Bayesian prior that $g>475 \mathrm{~m} \mathrm{~s}^{-2}$. We find $T_{\text {eff }}=533 \pm 8 \mathrm{~K}$, $f_{\text {sed }} \quad$ (cloudiness) $=4-5, \quad[\mathrm{M} / \mathrm{H}]=0.78 \pm 0.08, \quad R=0.95$ $\pm 0.06 R_{\mathrm{Jup}}$, and $\log (L)=-6.18 \pm 0.02 L_{\odot}$.

\subsubsection{Methane Absorption}

All of our models predict strong methane absorption at 1.66 and $3.3 \mu \mathrm{m}$, which is well matched by the photometry. Some warmer extrasolar planets (e.g., HR 8799 bcde and 2M1207 b) have effective temperatures where equilibrium chemistry models predict methane absorption (Marois et al. 2008; Barman et al. 2011a, 2011b). However, these planets show limited signs of methane absorption in near-infrared spectra (Patience et al. 2010; Barman et al. 2011a, 2015; Konopacky et al. 2013), or in mid-infrared SEDs (Hinz et al. 2010; Skemer et al. 2012, 2014b), indicating the presence of nonequilibrium chemistry in the $\mathrm{CH}_{4} \leftrightarrow \mathrm{CO}$ reaction network. For cooler planets, like GJ $504 \mathrm{~b}$, nonequilibrium chemistry is not expected to suppress methane absorption (Zahnle \& Marley 2014). The photometric upper limit to the brightness of GJ $504 \mathrm{~b}$ at $1.66 \mu \mathrm{m}(>20.62(3 \sigma)$; Janson et al. 2013) indicates the presence of methane opacity. Our narrow $L$-band photometry confirms that methane opacity is affecting the slope of the 3-4 $\mu \mathrm{m}$ SED in a way that is consistent with equilibrium chemistry models.

\section{SUMMARY AND CONCLUSIONS}

We obtained images of what is currently the coldest directly imaged exoplanet, GJ 504 b, in three narrow $L$-band filters, mapping out the $3.3 \mu \mathrm{m}$ methane fundamental absorption feature, and putting further constraints on the planet's basic physical properties. With a best-fit temperature of $550 \mathrm{~K}$, GJ $504 \mathrm{~b}$ is analogous to field brown dwarfs with a T spectral type. Indeed, the SED of GJ 504 b shows many similarities to a T-type brown dwarf: strong methane absorption features at 

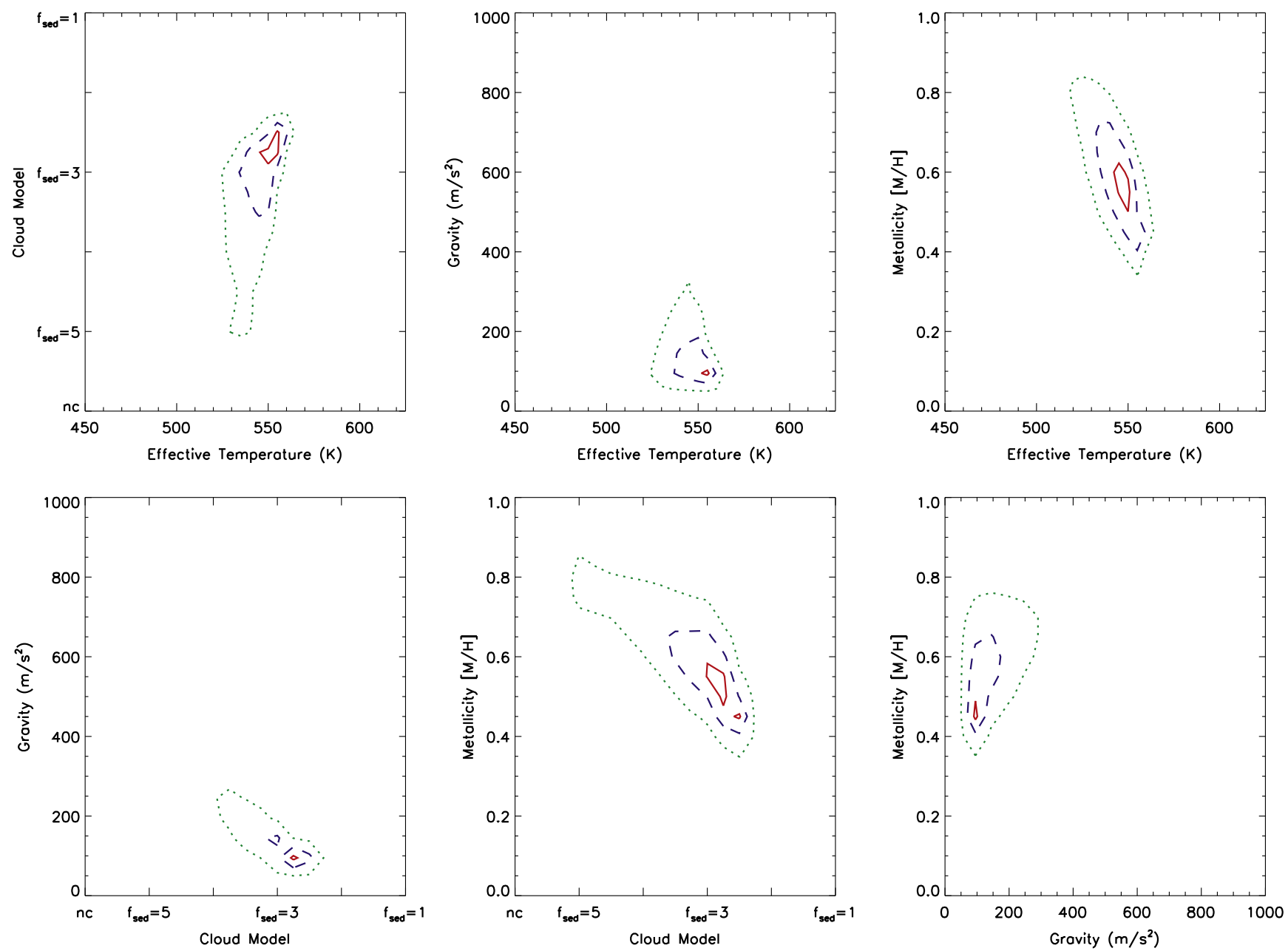

Figure 8. Contour plots of probability distributions for each pair of atmosphere model parameters. Models with $90 \%, 50 \%$, and $10 \%$ of the peak probability are shown with red solid contours, blue dashed contours, and green dotted contours, respectively.

1.66 and $3.3 \mu \mathrm{m}$, blue $J-H$ colors that imply a relative lack of clouds compared to red L dwarfs, and an SED that is reasonably well fit by a water-vapor-dominated spectrum. On the other hand, GJ 504 b's SED is different from any currently known field brown dwarf, particularly with regard to its unusually red $H-K_{s}$ colors.

We constructed a model grid with radius, temperature, metallicity, surface gravity, and cloud types as free parameters to try to explain GJ 504 b's unusual SED. We find $T_{\text {eff }}=544 \pm 10 \mathrm{~K}, f_{\text {sed }}$ (cloudiness) $=2-5, g<600 \mathrm{~m} \mathrm{~s}^{-2}$, $[\mathrm{M} / \mathrm{H}]=0.6 \pm 0.12, \quad R=0.96 \pm 0.07 R_{\mathrm{Jup}}, \quad$ and $\quad \log$ $(L)=-6.13 \pm 0.03 L_{\odot}$. If GJ 504 is young $(0.1-0.5 \mathrm{Gyr})$, Baraffe et al. (2003) models predict that the companion is 3-8 $M_{\text {jup }}$. If GJ 504 is old (3-6.5 Gyr), Baraffe et al. (2003) models predict that the companion is $19-30 M_{\text {jup }}$. Our estimate of the planet's surface gravity favors the low-mass interpretation, but not conclusively.

Of particular note, our planet atmosphere model requires a superstellar metallicity to explain GJ 504 b's complete SED, and particularly its $H-K_{s}$ colors shown in Figure 4. Various planet formation models predict that planet metallicities should differ from their host stars. Conversely, pairs of objects that form by binary fragmentation should have similar metallicities. Within the confines of our atmospheric models, this suggests that GJ $504 \mathrm{~b}$ formed like a planet, not like a binary companion. This result is independent of GJ 504 b's age and mass.

Additional photometry and spectroscopy at higher signal-tonoise ratio will further improve our ability to understand planets like GJ 504 b. In particular, observations over a broad wavelength range, including the mid-infrared $(\gtrsim 3 \mu \mathrm{m})$, can help break degeneracies in model parameters. Spectroscopy in the mid-infrared will be particularly valuable (Skemer et al. 2015). The James Webb Space Telescope (JWST) will discover and characterize a variety of new worlds (e.g., Beichman et al. 2010). The LEECH exoplanet imaging survey is searching for and characterizing cool exoplanets in the midinfrared right now, with the goal of improving our theoretical understanding of their atmospheres so that we can take full advantage of JWST's limited life span.

The authors thank the anonymous referee for a very helpful report. We thank the HiCIAO team and Masayuki Kuzuhara for providing filter curves for the HiCIAO methane filters. A.S. is supported by the National Aeronautics and Space Administration through Hubble Fellowship grant HSTHF2-51349 awarded by the Space Telescope Science Institute, which is operated by the Association of Universities for Research in Astronomy, Inc., for NASA, under contract NAS 5-26555. E. B. is supported by the Swiss National Science Foundation 
(SNSF). S.E., S.D., and A.L.M. acknowledge support from the "Progetti Premiali" funding scheme of the Italian Ministry of Education, University, and Research. C.V. is supported by NSF AST-1312305. LEECH is funded by the NASA Origins of Solar Systems Program, grant NNX13AJ17G. This material is based in part on work supported by the National Aeronautics and Space Administration under Agreement No. NNX15AD94G, Earths in Other Solar Systems, issued through the Science Mission Directorate interdivisional initiative Nexus for Exoplanet System Science. The Large Binocular Telescope Interferometer is funded by the National Aeronautics and Space Administration as part of its Exoplanet Exploration program. LMIRcam is funded by the National Science Foundation through grant NSF AST-0705296. This publication makes use of the Spex Prism Library Analysis Toolkit. This publication makes use of data products from the Wide-field Infrared Survey Explorer, which is a joint project of the University of California, Los Angeles, and the Jet Propulsion Laboratory/ California Institute of Technology, and NEOWISE, which is a project of the Jet Propulsion Laboratory/California Institute of Technology. WISE and NEOWISE are funded by the National Aeronautics and Space Administration.

\section{REFERENCES}

Ackerman, A. S., \& Marley, M. S. 2001, ApJ, 556, 872

Allard, N. F., Allard, F., \& Kielkopf, J. F. 2005, A\&A, 440, 1195

Amara, A., \& Quanz, S. P. 2012, MNRAS, 427, 948

Bailey, V. P., Hinz, P. M., Puglisi, A. T., et al. 2014, Proc. SPIE, 9148, 3

Baraffe, I., Chabrier, G., Barman, T. S., Allard, F., \& Hauschildt, P. H. 2003 , A\&A, 402, 701

Barman, T. S., Konopacky, Q. M., Macintosh, B., \& Marois, C. 2015, ApJ, 804,61

Barman, T. S., Macintosh, B., Konopacky, Q. M., \& Marois, C. 2011a, ApJ, 733, 65

Barman, T. S., Macintosh, B., Konopacky, Q. M., \& Marois, C. 2011b, ApJL, 735, L39

Beichman, C. A., Krist, J., Trauger, J. T., et al. 2010, PASP, 122, 162

Boley, A. C., \& Durisen, R. H. 2010, ApJ, 724, 618

Bonnefoy, M., Boccaletti, A., Lagrange, A.-M., et al. 2013, A\&A, 555, A107

Bonnefoy, M., Currie, T., Marleau, G.-D., et al. 2014, A\&A, 562, A111

Bonnefoy, M., Lagrange, A.-M., Boccaletti, A., et al. 2011, A\&A, 528, L15

Carlberg, J. K., Cunha, K., Smith, V. V., \& Majewski, S. R. 2012, ApJ, 757, 109

Castelli, F., \& Kurucz, R. L. 2004, arXiv:astro-ph/0405087

Chauvin, G., Lagrange, A.-M., Dumas, C., et al. 2004, A\&A, 425, L29

Cutri, R. M., et al. 2013, yCat, 2328, 0

Desidera, S., Gratton, R. G., Lucatello, S., \& Claudi, R. U. 2006, A\&A, 454,581

Desidera, S., Gratton, R. G., Scuderi, S., et al. 2004, A\&A, 420, 683

Dupuy, T. J., \& Liu, M. C. 2012, ApJS, 201, 19

Edvardsson, B., Andersen, J., Gustafsson, B., et al. 1993, A\&A, 275, 101

Esposito, S., Riccardi, A., Pinna, E., et al. 2011, Proc. SPIE, 8149, 814902

Faherty, J. K., Rice, E. L., Cruz, K. L., Mamajek, E. E., \& Núñez, A. 2013, AJ, 145,2

Fergus, R., Hogg, D. W., Oppenheimer, R., Brenner, D., \& Pueyo, L. 2014, ApJ, 794, 161

Fischer, D. A., \& Valenti, J. 2005, ApJ, 622, 1102

Fortney, J. J., Marley, M. S., \& Barnes, J. W. 2007, ApJ, 659, 1661

Fortney, J. J., Marley, M. S., Saumon, D., \& Lodders, K. 2008, ApJ, 683,1104

Fuhrmann, K., \& Chini, R. 2015, ApJ, 806, 163

Gauza, B., Béjar, V. J. S., Pérez-Garrido, A., et al. 2015, ApJ, 804, 96

Gonzalez, G., Carlson, M. K., \& Tobin, R. W. 2010, MNRAS, 403, 1368
Hinz, P., Arbo, P., Bailey, V., et al. 2012, Proc. SPIE, 8445, 84450U

Hinz, P. M., Rodigas, T. J., Kenworthy, M. A., et al. 2010, ApJ, 716, 417

Janson, M., Brandt, T. D., Kuzuhara, M., et al. 2013, ApJL, 778, L4

Janson, M., Carson, J., Thalmann, C., et al. 2011, ApJ, 728, 85

Kidger, M. R., \& Martín-Luis, F. 2003, AJ, 125, 3311

Konopacky, Q. M., Barman, T. S., Macintosh, B. A., \& Marois, C. 2013, Sci, 339, 1398

Kuzuhara, M., Tamura, M., Kudo, T., et al. 2013, ApJ, 774, 11

Lafrenière, D., Marois, C., Doyon, R., Nadeau, D., \& Artigau, É. 2007, ApJ, 660,770

Leisenring, J. M., Skrutskie, M. F., Hinz, P. M., et al. 2012, Proc. SPIE, 8446, $84464 \mathrm{~F}$

Liu, M. C., Magnier, E. A., Deacon, N. R., et al. 2013, ApJL, 777, L20

Lloyd-Hart, M. 2000, PASP, 112, 264

Lodders, K. 1999, ApJ, 519, 793

Lodders, K. 2002, ApJ, 577, 974

Lodders, K., \& Fegley, B. 2002, Icar, 155, 393

Lodders, K., \& Fegley, B., Jr. 2006, in Astrophysics Update 2, ed. J. W. Mason (New York: Springer), 1

Macintosh, B., Graham, J. R., Barman, T., et al. 2015, Sci, 350, 64

Maire, A.-L., Skemer, A. J., Hinz, P. M., et al. 2015, A\&A, 576, A133

Maldonado, J., Eiroa, C., Villaver, E., Montesinos, B., \& Mora, A. 2012, A\&A, 541, A40

Mamajek, E. E., \& Hillenbrand, L. A. 2008, ApJ, 687, 1264

Marley, M. S., Saumon, D., Cushing, M., et al. 2012, ApJ, 754, 135

Marois, C., Lafrenière, D., Doyon, R., Macintosh, B., \& Nadeau, D. 2006, ApJ, 641,556

Marois, C., Macintosh, B., Barman, T., et al. 2008, Sci, 322, 1348

Marois, C., Zuckerman, B., Konopacky, Q. M., Macintosh, B., \& Barman, T. 2010, Natur, 468, 1080

Mishenina, T. V., Soubiran, C., Kovtyukh, V. V., \& Korotin, S. A. 2004, A\&A, 418, 551

Morley, C. V., Fortney, J. J., Marley, M. S., et al. 2012, ApJ, 756, 172

Morley, C. V., Marley, M. S., Fortney, J. J., et al. 2014, ApJ, 787, 78

Moses, J. I., Madhusudhan, N., Visscher, C., \& Freedman, R. S. 2013, ApJ, 763,25

Nayakshin, S. 2015, MNRAS, 448, L25

Patience, J., King, R. R., de Rosa, R. J., \& Marois, C. 2010, A\&A, 517, A76

Patience, J., King, R. R., De Rosa, R. J., et al. 2012, A\&A, 540, A85

Podolak, M., Pollack, J. B., \& Reynolds, R. T. 1988, Icar, 73, 163

Pollack, J. B., Podolak, M., Bodenheimer, P., \& Christofferson, B. 1986, Icar, 67, 409

Ramírez, I., Allende Prieto, C., \& Lambert, D. L. 2013, ApJ, 764, 78

Rieke, G. H., Blaylock, M., Decin, L., et al. 2008, AJ, 135, 2245

Skemer, A. J., Close, L. M., Szúcs, L., et al. 2011, ApJ, 732, 107

Skemer, A. J., Hinz, P., Esposito, S., et al. 2014a, Proc. SPIE, 9148, 0

Skemer, A. J., Hinz, P., Montoya, M., et al. 2015, Proc. SPIE, 9605, 96051D

Skemer, A. J., Hinz, P. M., Esposito, S., et al. 2012, ApJ, 753, 14

Skemer, A. J., Marley, M. S., Hinz, P. M., et al. 2014b, ApJ, 792, 17

Skrutskie, M. F., Jones, T., Hinz, P., et al. 2010, Proc. SPIE, 7735, 77353H

Skrutskie, M. F., Wilson, J., Nelson, M., et al. 2014, in AAS/Division for Planetary Sciences Meeting Abstracts 46, 41815

Soderblom, D. R. 2010, in IAU Symp. 268, Light Elements in the Universe, ed. C. Charbonnel et al. (Cambridge: Cambridge Univ. Press), 359

Soummer, R., Pueyo, L., \& Larkin, J. 2012, ApJL, 755, L28

Takeda, Y. 2007, PASJ, 59, 335

Tamura, M. 2009, in AIP Conf. Ser. 1158, ed. T. Usuda, M. Tamura, \& M. Ishii (Melville, NY: AIP), 11

Thalmann, C., Carson, J., Janson, M., et al. 2009, ApJL, 707, L123

Valenti, J. A., \& Fischer, D. A. 2005, ApJS, 159, 141

van Leeuwen, F. 2007, A\&A, 474, 653

Visscher, C. 2012, ApJ, 757, 5

Visscher, C., Lodders, K., \& Fegley, B., Jr. 2006, ApJ, 648, 1181

Visscher, C., Lodders, K., \& Fegley, B., Jr. 2010, ApJ, 716, 1060

Yurchenko, S. N., Tennyson, J., Bailey, J., Hollis, M. D. J., \& Tinetti, G. 2014, PNAS, 111,9379

Zahnle, K. J., \& Marley, M. S. 2014, ApJ, 797, 41 